\title{
Implicit Power Motivation Predicts Men's Testosterone Changes and Implicit Learning in a Contest Situation
}

\author{
Oliver C. Schultheiss*,1 and Wolfgang Rohde† \\ *Department of Psychology, University of Potsdam, P. O. Box 601553, 14415 Potsdam, \\ Germany; and Institute of Experimental Endocrinology at the Charité Hospital, \\ Humboldt University, Schuhmannstr. 20/21, 10117 Berlin, Germany
}

Received March 23, 2001; revised July 19, 2001; accepted August 21, 2001

\begin{abstract}
This study tested the hypothesis that implicit power motivation moderates men's testosterone responses to victory or defeat in a contest situation. It also explored to what extent postvictory testosterone increases are associated with enhanced implicit learning of behavior instrumental for winning a contest. Salivary testosterone levels were assessed in $\mathbf{6 6}$ male adults several times before and after a contest whose outcome (winning or losing against a competitor on an implicit learning task) was varied experimentally. Among participants low in activity inhibition, a measure of impulse control, the power motive was a significant positive predictor of testosterone increases (15 min postcontest; $r=0.71, P=$ $0.01)$ and implicit learning $(r=0.68, P<0.05)$ after a victory, whereas it was a significant negative predictor of implicit learning $(r=-0.58, P=0.01)$ but not of testosterone increases ( $r=-0.08, n s)$ after a defeat. Moreover, among participants low in activity inhibition testosterone increases were associated with enhanced implicit learning $(r=0.38, P<0.05)$ and there was statistical evidence that in winners testosterone increases mediated the effect of power motivation on implicit learning. Participants high in activity inhibition did not display this pattern of results. $\odot 2002$ Elsevier Science (USA)

Key Words: testosterone; implicit power motive; motivation; activity inhibition; dominance contest; implicit learning.
\end{abstract}

The steroid hormone testosterone has been implicated in social dominance and aggressive behavior in a wide variety of species, including humans (Bern-

\footnotetext{
${ }^{1}$ To whom correspondence and reprint requests should be addressed at present address: Department of Psychology, 525 East University Ave., University of Michigan, Ann Arbor, Michigan 48109. Fax: +1 734647 9440. E-mail: oschult@umich.edu.
}

stein, Gordon, and Rose, 1983; Mazur and Booth, 1998; Monaghan and Glickman, 1992). Specifically, many studies document rising testosterone levels in response to a dominance success but unchanged or even declining testosterone levels after a defeat (Bernstein et al., 1983; Mazur and Booth, 1998). Mazur (1985) has speculated that postvictory testosterone surges, which can be observed within minutes and up to some hours after a dominance success, may serve to reinforce behavior that was instrumental for achieving the victory. This hypothesis has gained plausibility by recent findings that document rewarding (e.g., Packard, Schroeder, and Alexander, 1998) and mood-enhancing (e.g., Rabkin, Wagner, and Rabkin, 1996) effects of testosterone administration.

In humans, empirical support for a direct effect of winning and losing a competition on testosterone is equivocal. While some researchers report higher postcontest testosterone levels in winners than in losers (e.g., Gladue, Boechler, and McCaul, 1989; Mazur and Lamb, 1980; McCaul, Gladue, and Joppa, 1992), others have failed to obtain such a difference (e.g., GonzalezBono, Salvador, Serrano, and Ricarte, 1999; Mazur, Susman, and Edelbrock, 1997; Schultheiss, Campbell, and McClelland, 1999; Suay et al., 1999), thus raising doubts about the efficacy of situational outcomes to affect testosterone levels directly and reliably.

Schultheiss et al. (1999) have therefore recently argued that motivational dispositions may moderate the impact of situational outcomes such as winning or losing a competition on testosterone changes (for related arguments, see Berman, Gladue, and Taylor, 1993). In support of this notion, they reported evidence for a moderating role of the implicit power motive, the nonconscious disposition to experience 
having impact on others as rewarding (McClelland, 1975; Winter, 1973), on men's testosterone responses to the outcome of a dominance contest. Specifically, they found that individuals high in personalized power motivation, a subtype of the power motive that aims at having impact through assertive behavior (cf. McClelland, 1975), responded with increased testosterone levels to beating an opponent in a speed-based competitive task but not to a defeat. In contrast, individuals low in personalized power motivation or high in socialized power motivation, a subtype of the power motive that aims at having impact through prosocial behavior, did not respond with a testosterone increase to winning or losing the contest.

In the present study, our goal was to replicate the findings reported by Schultheiss et al. (1999) in a larger sample and to explore whether postvictory testosterone increases are indeed reinforcing, as suggested by Mazur (1985). We reasoned that if beating an opponent is rewarding for high-power individuals and if testosterone increases are associated with this pleasurable impact experience, then (a) higher levels of power motivation should be related to better learning of behavior that is instrumental for winning the contest and (b) testosterone increases should be related to, and presumably facilitate, instrumental learning in highpower winners.

Because we expected these relationships to emerge for individuals with a personalized power motive but not for those with a socialized power motive in our contest paradigm, we assessed participants' level of activity inhibition, a measure of dispositional impulse control, in addition to their implicit power motive. Previous research has established that a strong power motive paired with low inhibition leads individuals to seek impact in a personalized, assertive manner, whereas a strong power motive paired with high inhibition is typically associated with socialized and controlled ways of seeking impact (McClelland, 1975, 1987; Schultheiss and Brunstein, in press). Thus, we expected an interaction effect of contest outcome and participants' power motive and inhibition levels on testosterone changes and implicit learning.

To measure the effects of personality variables and contest outcome on instrumental learning, we had participants compete on an implicit learning task during the contest and subsequently measured how well they had learned a repetitive visuomotor pattern embedded in that task. Implicit learning has been defined as nonintentional and nonconscious learning (Reber, 1989) and has been shown to be influenced by personality dispositions, reward, and punishment (Corr,
Pickering, and Gray, 1997). We explored to what extent increases in testosterone were associated with better implicit learning and whether effects of power motivation and contest outcome on implicit learning could be explained by their effects on testosterone changes.

Finally, we included multiple hormone measurements before and after the contest to study the time course of the effects of power motivation and contest outcome on testosterone in some detail. We tested our hypotheses in a sample of male college students because salivary testosterone levels are about 3- to 10fold higher, and thus easier to measure, in adult men than in adult women and also because testosterone may not be related to dominant and assertive behavior in women as strongly as it is in men (Mazur and Booth, 1998).

\section{METHOD}

Participants. Sixty-six male students of a vocational college in Potsdam, Germany, who were $23.83 \pm 0.41$ years old, nonsmokers, had a body mass index of $22.37 \pm 0.31$, and had refrained from eating and oral hygiene for at least $1 \mathrm{~h}$ before arriving at the laboratory, participated pairwise in sessions lasting about $150 \mathrm{~min}$ for a remuneration of 35 Deutschemarks $(\sim \$ 15)$. Differences in participants' hormone levels due to diurnal variations were minimized by holding sessions only at 1:45 and 4:00 PM.

Design. The study was based on a Contest Outcome $\times$ Power Motive $\times$ Inhibition design. Contest outcome was varied by having one participant in each dyad win $(N=33)$ and the other lose $(N=33)$ a dominance contest. Participants were assigned randomly to conditions. Participants' power motive and inhibition levels were assessed with a Picture Story Exercise (PSE). Dependent variables were testosterone changes and implicit learning.

Procedure and condition. Each session was run by a male experimenter and consisted of a precontest, a contest, and a postcontest phase. In the precontest phase, participants provided a saliva sample (T1, at 0 min) and then completed a PSE and worked on 8 Number Tracking Test (NTT) forms (four sequence and four random forms in alternating order; see below) for $25 \mathrm{~s}$ each. Next, the experimenter announced that participants would now compete against each other on a contest based on the NTT. Participants then provided a second saliva sample (T2, at $60 \mathrm{~min}$ ), listened to a tape-recorded goal imagery exercise vividly 
describing the course of the ensuing contest from the winner's perspective (cf. Schultheiss, 2001), and provided a third saliva sample (T3, at $75 \mathrm{~min}$ ). During the contest phase, participants competed against each other on 12 NTT forms (6 sequence and 6 random forms in alternating order, starting with a sequence form). The participant finishing first by reaching a highlighted end number on a given form was instructed to say "Done!" and the other participant had to stop immediately and was thus prevented from finishing his form. Undetectable to participants, the distance between the start number and the end number was 20 numbers shorter on "winning" than on "losing" NTT forms. "Winning" and "losing" forms were administered to participants in such a way that the winner won eight times (on forms 1, 4, 6, 7, and 9 through 12) and the loser only four times (on forms 2 , 3,5 , and 8 ). In fact, winners did not significantly differ from losers in their performance on either form type or both types combined. At the beginning of the postcontest phase, participants collected a fourth saliva sample (T4, at $95 \mathrm{~min}$ ) and a fifth saliva sample (T5, at $110 \mathrm{~min}$ ). They then worked on another 8 NTT forms (4 sequence and 4 random forms in alternating order) for $25 \mathrm{~s}$ each and collected a final saliva sample (T6, at $130 \mathrm{~min}$ ) while providing basic biographical information about themselves and taking an implicit learning awareness test. They were fully debriefed about the hypotheses underlying the study and the manipulations employed.

Picture story exercise. The PSE was administered to participants using standard instructions described in Smith (1992). It consisted of five pictures that have been widely used in research on implicit motives. Participants had $5 \mathrm{~min}$ to write each story. Stories were later coded for power motive imagery according to Winter's (1994) Manual for Scoring Motive Imagery in Running Text. Two trained scorers, who had previously attained at least $85 \%$ agreement with training materials prescored by experts and contained in the Manual, independently coded each participant's stories. Their interrater reliability across all stories was $84 \%$, as estimated by the index of concordance, and scoring disagreements were resolved in joint sessions. Both scorers also determined participants' level of activity inhibition by counting the frequency of the negation not (German, nicht) in participants' stories. An index of concordance of over $98 \%$ indicated that both scorers detected this word with high reliability. Scoring disagreements were resolved by averaging counts across scorers. Participants wrote $388 \pm 10$ words, and their scores were $3.67 \pm 0.25$ for power motivation and $3.32 \pm 0.25$ for activity inhibition. Participants' power and inhibition scores were corrected by regression for PSE protocol length, which correlated significantly with power and inhibition $\left(P_{\mathrm{s}}<\right.$ $0.05)$, and the residuals were converted to $Z$ scores. These standardized scores were used as quantitative predictor variables in further analyses.

Salivary testosterone measurement. Sample collections took about $5 \mathrm{~min}$ each and were spaced at least $10 \mathrm{~min}$ apart, thus providing ample time for salivary steroid levels to approach equilibrium with free steroid levels in serum. At each sampling point, participants used a fresh sugar-free chewing gum to collect between 2 and $10 \mathrm{~mL}$ of saliva (mean volumes ranged between 4.07 and $5.40 \mathrm{~mL}$ across all six collections) in a sterile polypropylene vial and then removed the chewing gum (Dabbs, 1991). Vials were closed and frozen immediately at the end of each data collection session. Samples were freed from mucopolysaccarides and proteins by several freeze-thaw cycles with subsequent centrifugation. Salivary testosterone levels were determined by solid-phase ${ }^{125} \mathrm{I}$ radioimmunoassays (Coat-A-Count, Diagnostic Products Corp., Biermann, Bad Nauheim, Germany), using the protocol described by Campbell, Schultheiss, and McClelland (1999). Intra- and interassay CVs were $13 \%$, averaged across three assays. Sensitivity was at 8 $\mathrm{pg} / \mathrm{ml}$.

Number tracking test. On the NTT, participants were required to connect a sequence of consecutive ascending numbers (1-2-3-4-...), which were surrounded by distractor numbers and arranged in a $15 \times 15$ matrix, as fast as possible with a pen. The highest valid consecutive number a participant reached on a form, which could either be a highlighted target number during the contest or a lower number if he was stopped by his competitor or the experimenter, represented his performance on that form. Sequence NTT forms contained a repetitive visuomotor pattern consisting of five pen movements in fixed directions, alternating with portions of five pen movements in random directions. The pattern was the same across different sequence forms. On random NTT forms, stroke directions between consecutive numbers were entirely random. Thus, sequence forms contained portions that could be learned across forms, whereas on random forms the next pen stroke could not be predicted from the preceding one. All possible stroke directions (up, up right, right, down right, down, down left, left, up left) were represented with approximately equal likelihood and the frequency of each stroke direction on the sequence forms did not differ 
from its counterpart on the random forms, $T^{2}(8,7)=$ $1.57, n s$. Therefore, whatever learning occurred could not be attributed to some pen movements occurring more often than others on the sequence forms than on the random forms but is likely to represent more complex sequence learning (cf. Reed and Johnson, 1994). For the pre- and postcontest administrations of the NTT, all forms were randomized within and across assessments so that no two participants worked on a set of the same four sequence (or random) forms before or after the contest. Whether participants started with a sequence or a random form on both the pre- and the postcontest administrations was balanced across participants.

Awareness test. Being queried about their observations at the end of the session, no participant mentioned anything related to a regularity or pattern featured in the NTT forms. When participants were asked to draw a frequently occurring NTT pattern with a pen in a dot matrix, only one reproduced the visuomotor pattern characteristic of the sequence forms, but he did not check this pattern on the subsequent forced-choice test. A final forced-choice test featured nine different five-stroke connection patterns and participants had to identify one as the repetitive pattern. All nine patterns had been taken from the original NTT forms participants had worked on, but only one represented the repetitive visuomotor pattern of the sequence forms. Only three participants correctly identified the pattern, which is even less than would be expected by chance. Thus, participants appeared to be unaware of the fact that half of the NTT forms contained a repetitive sequence of number connections.

Statistical procedures. Contest outcome was coded 1 for winners and -1 for losers. All analyses were conducted with SPSS 9.0 and involved regression and correlation analysis, analysis of variance, and univariate and multivariate $t$ tests. When higher order effects were tested for significance, all lower order effects were controlled for first. Descriptive statistics are given as means \pm SEM and effect size estimates as $R^{2}$. An $\alpha$ level of 0.05 was employed in all analyses.

\section{RESULTS}

Testosterone. For T1 through T6, participants' salivary testosterone levels were $111 \pm 4,108 \pm 4,110 \pm$ 4, $108 \pm 4,105 \pm 4$, and $102 \pm 4 \mathrm{pg} / \mathrm{mL}$. Intercorrelations between subsequent testosterone measurements were highly stable, with coefficients ranging between 0.84 and 0.93 . Winners did not significantly differ from losers in their pre- or postcontest testosterone levels. We did not detect any separate or conjoint effects of participants' power motive and activity inhibition on baseline testosterone levels (T1) or their responses to the contest instruction (T2) or the goal imagery exercise (T3), even after controlling for preceding measurements. To evaluate our main hypothesis, namely, that high-power motivation predicts postcontest testosterone increases in winners low in inhibition, we conducted regression analyses with testosterone changes from $\mathrm{T} 3$ to $\mathrm{T} 4, \mathrm{~T} 5$, and T6 as dependent variables. Our use of change scores seems justified because (a) testosterone levels were so stable across measurements that absolute values were less meaningful than relative changes from one measurement to the next, (b) they provided a straightforward way to plot results, and (c) all significance levels remained unchanged when we tested postcontest testosterone controlled for precontest testosterone, which indicates that our results are not artifactual. While the hypothesized Contest Outcome $\times$ Power Motive $\times$ Inhibition interaction failed to become significant for testosterone changes immediately after the contest (T3 to T4) and $30 \mathrm{~min}$ after the contest (T3 to T6), it was a significant unique predictor of testosterone changes from $\mathrm{T} 3$ to $\mathrm{T} 5$, that is, $15 \mathrm{~min}$ after the contest, $B=$ $-4.38, S E=1.99, t(58)=-2.19, P<0.05$ (full model $R^{2}=0.213$ ). Follow-up analyses revealed that this effect was mainly due to participants low in inhibition (with a $Z$ score $\leq-0.15$ ) whose power motive significantly predicted testosterone increases after winning the contest, $r=0.71, P=0.01$, but not after losing, $r=-0.08$, ns (for the interaction, $B=6.67$, $S E=3.02, t(29)=2.21, P<0.05$; full model $R^{2}=$ 0.229). This Contest Outcome $\times$ Power Motive interaction did not become significant for participants high in inhibition (with a $Z$ score $>-0.15$ ). Figure 1 illustrates these findings.

Implicit learning. To test whether the NTT measured sequence learning above and beyond performance changes not related to learning, we first computed sum scores for the 4 precontest sequence forms, the 4 precontest random forms, the 4 postcontest sequence forms, and the 4 postcontest random forms and then subjected these to a repeated-measures ANOVA with form (sequence vs random) and time (precontest vs postcontest) as within-subjects factors. A highly significant Form $\times$ Time interaction, $F(1$, $65)=12.56, P<0.001$, indicated that while participants did not significantly differ in their precontest performance on sequence forms $(120.53 \pm 3.10)$ and 


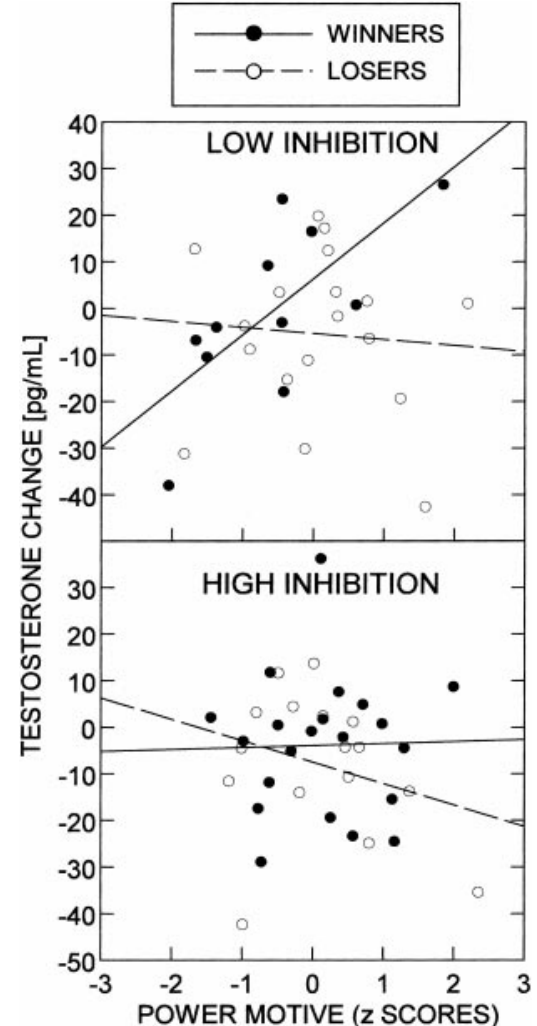

FIG. 1. Changes in salivary testosterone from T3 (immediately before the contest) to $\mathrm{T} 5$ (15 min postcontest) as a function of winning or losing the contest and implicit power motivation for participants low $(Z$ score $\leq-0.15)$ or high $(Z$ score $>-0.15)$ in activity inhibition.

random forms $(120.26 \pm 2.85)$, they showed stronger pre-postcontest increases on sequence forms, $t(65)=$ 15.22, $P<0.001$, than on random forms, $t(65)=$ 13.12, $P<0.001$, and thus performed significantly better on postcontest sequence forms (152.02 \pm 2.92$)$ than on postcontest random forms (144.06 \pm 2.51$)$, $t(65)=5.71, P<0.001$. We interpreted the postcontest performance advantage on sequence forms as an indication that participants had learned the visuomotor sequence embedded in those forms. Since we were interested in whether the extent of learning could be accounted for by our predictors, we then created an index of implicit learning by subtracting each participant's performance on postcontest random forms from his performance on postcontest sequence forms (cf. Reed and Johnson, 1994). Regression analyses indicated that the predicted Contest Outcome $\times$ Power Motive $\times$ Inhibition interaction on implicit learning was significant, $B=-3.85, S E=1.44, t(65)=$ $-2.68, P=0.01$ (full model $R^{2}=0.160$ ). As illus- trated in Fig. 2, this effect was due to a strong positive correlation between power motivation and learning among low-inhibition winners, $r=0.68, P<0.05$, and a strong negative correlation of power motivation and learning among low-inhibition losers, $r=-0.58$, $P=0.01$ (for the interaction, $B=6.24, S E=1.61$, $t(28)=3.87, P<0.001$; full model $\left.R^{2}=0.382\right)$. The interaction between contest outcome and power motivation did not become significant for high-inhibition participants.

Mediation analyses. While testosterone changes (from T3 to T5) were not significantly associated with implicit learning in the overall sample, $r=0.08$, they were among low-inhibition participants, $r=0.38$, $P<0.05$, with the correlation being slightly stronger for winners $(r=0.41)$ than for losers $(r=0.36)$. Was there any evidence that testosterone increases mediated the effect of power motivation on implicit learning among low-inhibition winners? One way to answer this question is to examine whether the effect of

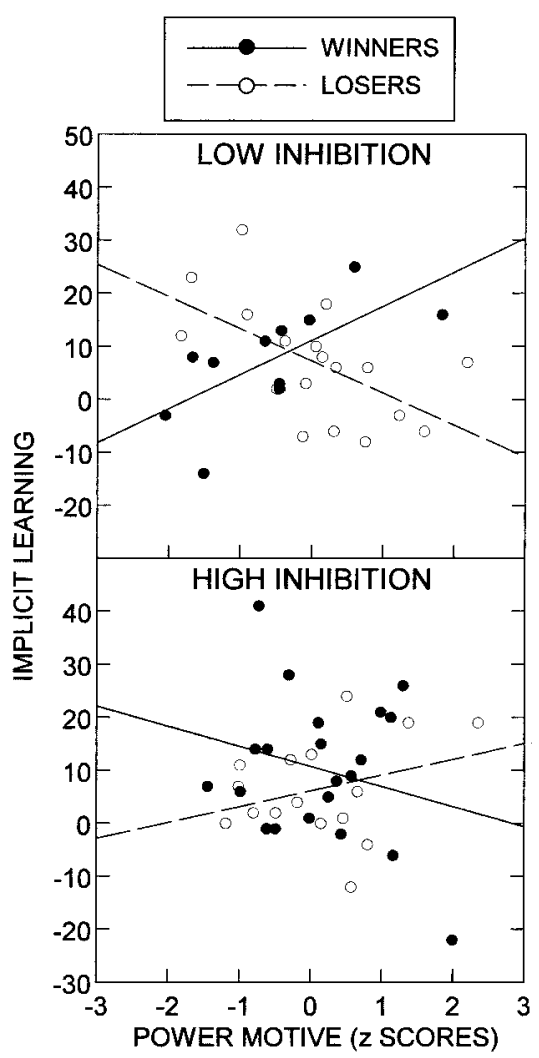

FIG. 2. Implicit learning (postcontest performance on sequence NTT forms minus postcontest performance on random NTT forms) as a function of winning or losing the contest and implicit power motivation for participants low $(Z$ score $\leq-0.15)$ or high $(Z$ score $>-0.15)$ in activity inhibition. 
power motivation on instrumental learning remains significant after controlling for testosterone changes. If testosterone changes are indeed responsible for the power motive-implicit learning relationship, then the power motive-implicit learning correlation should no longer be significant after controlling for testosterone changes (cf. Baron and Kenny, 1986; McCaul et al., 1992). This was confirmed by our analyses: the power motive did not have a significant effect on implicit learning after partialling for testosterone changes, $P>$ 0.05. Importantly, the power motive remained a significant $(P<0.05)$ predictor of testosterone after partialling for implicit learning, which suggests that high-power participants' testosterone increases cannot have been due to their enhanced implicit learning.

\section{DISCUSSION}

The results of this study replicate those reported by Schultheiss et al. (1999) in a larger sample with a different sociocultural background and thus support the hypothesis that implicit power motivation plays a moderating role in individuals' testosterone responses to winning a contest. Specifically, we found that higher levels of power motivation predicted stronger postcontest testosterone increases $15 \mathrm{~min}$ after a victory but not after a defeat. Consistent with the notion that beating an opponent in a contest is a more attractive incentive for individuals with a personalized power motive than for those with a socialized power motive, this effect emerged only for individuals low but not for those high in activity inhibition, a measure of impulse control.

Notably, we did not detect any direct effect of winning and losing on postcontest testosterone changes, independently of power motivation and inhibition. Although we cannot rule out that we might have obtained such an effect with a more precise assay (but see Gonzalez-Bono et al., 1999, and Mazur et al., 1997, for similar null findings with highly precise assays), we believe that this finding reinforces Schultheiss et al.'s (1999) conclusion that situational factors by themselves are often insufficient to predict testosterone changes and that their interplay with motivational dispositions is more important.

In contrast to the earlier study by these researchers, we did not find that fantasizing about a victory affects subsequent testosterone changes. We suggest that the failure to replicate this effect may be due in part to our German sample being significantly lower in overall power motivation $(9.46 \pm 0.60$ power scores per 1000 words), and thus less sensitive to power-related incentives and outcomes, than Schultheiss et al.'s United States sample $(12.30 \pm 0.55$ power scores per 1000 words), $P<0.001$. These between-sample differences in power motivation may also explain the higher stability of testosterone measurements and the overall smaller effects of predictors on testosterone in our present study as compared to Schultheiss et al.'s (1999) study. Alternatively, the goal imagery exercise used in the present study may have been less effective and thus less power motivation-arousing than the one used in the earlier study.

Importantly, we found that for low-inhibition participants, the power motive and contest outcome had strong effects on implicit learning of behavior that preceded a victory or defeat. In winners, high levels of power motivation predicted enhanced learning of a visuomotor sequence embedded in the contest task, whereas in losers, high levels of power motivation predicted impaired learning of this sequence. These results suggest that for noninhibited power-motivated individuals, having impact by beating an opponent is indeed a rewarding experience and behavior leading to this outcome will thus become more energized, whereas being beaten by another person is aversive and behavior associated with this outcome will not be invested with motivational energy in the future. Notably, participants did not seem to be aware of the fact that they actually learned a sequence during the contest, which demonstrates that the implicit power motive in conjunction with situational factors can shape individuals' behavior without their knowledge.

We also found that testosterone increases were correlated with implicit learning among low-inhibition participants and that in winners, these testosterone increases may have been responsible for the effect of higher levels of power motivation on enhanced implicit learning. While these findings are consistent with rewarding effects of testosterone in animals (e.g., Packard et al., 1998) and support our hypothesis that testosterone increases may be associated with power motivation reward and reinforcement in humans, they should be interpreted with some caution. First, our evidence for such a reinforcing effect of testosterone increases on learning is based on a relatively small subsample $(N=11)$ of low-inhibition contest winners and is of a correlational nature only. No strong causal inferences can therefore be drawn at this point. Second, a testosterone surge $15 \mathrm{~min}$ postcontest may be too late to have rewarding properties and reinforce behavior. Note, however, that steroid peaks in blood precede steroid peaks in saliva by about 3 to $5 \mathrm{~min}$ (Riad-Fahmy, Read, Walker, Walker, and 
Griffiths, 1987) and that victory or defeat, either alone or in combination with personality dispositions, has been found to influence salivary testosterone levels immediately after a contest in earlier research (e.g., Gladue et al., 1989; Schultheiss et al., 1999). Thus, high-power winners low in inhibition may have reached peak levels of testosterone in blood earlier than 15 min postcontest in our study, although we do not know how much earlier. Third, the mechanism for Mazur's (1985) hypothesized reinforcing effect of postvictory testosterone surges is presently unknown. It is possible that the association between testosterone surges and implicit learning among low-inhibition winners may have been mediated by fast, nongenomic effects of testosterone on dopaminebased incentive learning or by slow-acting genomic effects on memory consolidation, or both (cf. Flood, Morley, and Roberts, 1992; Packard et al., 1998; Moore and Evans, 1999). Alternatively, the correlation of testosterone increases with implicit learning in low-inhibition winners may be spurious and reflect the operation of a more central mechanism underlying both the testosterone and the implicit learning effects observed in our study. These issues need to be addressed by bringing testosterone release and its effects on the brain under experimental control by, for instance, blocking the release of testosterone in the testes and adrenals or inhibiting its binding to brain sites involved in reward and reinforcement.

The present findings leave open the question whether power motivation will predict testosterone and learning responses to winning or losing a dominance contest in women as it does in men. Likewise, we did not address the issue of whether there may be instances in which the power motive predicts testosterone release and instrumental learning in response to having impact, or failing to have impact, in highinhibition individuals, as may be the case in situations that offer an opportunity to exert prosocial influence on others (e.g., Schultheiss and Brunstein, in press). Still, we would like to emphasize that our findings show for the first time that (a) an implicit motive in combination with situational outcomes predicts instrumental learning and that by this criterion having impact is indeed reinforcing for high-power individuals and (b) implicit learning is associated with hormonal changes in some individuals.

\section{ACKNOWLEDGMENTS}

We thank Alexander Gerlach and Martin Krippl for their help in collecting the data and Katharina Thiele for scoring the PSE stories.
This work was supported by a Deutsche Forschungsgemeinschaft research stipend to Oliver C. Schultheiss (SCHU 1210/1-2, 1-3).

\section{REFERENCES}

Baron, R. M., and Kenny, D. A. (1986). The moderator-mediator variable distinction in social psychological research: Conceptual, strategic, and statistical considerations. J. Pers. Soc. Psychol. 51, 1173-1182.

Berman, M., Gladue, B., and Taylor, S. (1993). The effects of hormones, Type A behavior pattern, and provocation on aggression in men. Motiv. Emotion 17, 125-138.

Bernstein, I. S., Gordon, T. P., and Rose, R. M. (1983). The interaction of hormones, behavior, and social context in nonhuman primates. In B. B. Svare (Ed.), Hormones and Aggressive Behavior, pp. 535-561. Plenum, New York.

Campbell, K. L., Schultheiss, O. C., and McClelland, D. C. (1999). A necessary adjustment of protocol for use of DPC Coat-A-Count total testosterone assay with saliva. Clin. Biochem. 32(1), 83-85.

Corr, P. J., Pickering, A. D., and Gray, J. A. (1997). Personality, punishment, and procedural learning: a test of J.A. Gray's anxiety theory. J. Pers. Soc. Psychol. 73(2), 337-344.

Dabbs, J. M. (1991). Salivary testosterone measurements: Collecting, storing, and mailing saliva samples. Physiol. Behav. 49, 815-817.

Flood, J. F., Morley, J. E., and Roberts, E. (1992). Memory-enhancing effects in male mice of pregnenolone and steroids metabolically derived from it. Proc. Natl. Acad. Sci. USA 89(5), 1567-1571.

Gladue, B. A., Boechler, M., and McCaul, K. D. (1989). Hormonal response to competition in human males. Aggr. Behav. 15, 409422.

Gonzalez-Bono, E., Salvador, A., Serrano, M. A., and Ricarte, J. (1999). Testosterone, cortisol, and mood in a sports team competition. Horm. Behav. 35, 55-62.

Mazur, A. (1985). A biosocial model of status in face-to-face primate groups. Soc. Forces 64, 377-402.

Mazur, A., and Booth, A. (1998). Testosterone and dominance in men. Behav. Brain Sci. 21, 353-397.

Mazur, A., and Lamb, T. A. (1980). Testosterone, status, and mood in human males. Horm. Behav. 14, 236-246.

Mazur, A., Susman, E. J., and Edelbrock, S. (1997). Sex differences in testosterone response to a video game contest. Evol. Hum. Behav. 18, 317-326.

McCaul, K. D., Gladue, B. A., and Joppa, M. (1992). Winning, losing, mood, and testosterone. Horm. Behav. 26, 486-504.

McClelland, D. C. (1975). Power: The Inner Experience. Irvington, New York.

McClelland, D. C. (1987). Human Motivation. Cambridge Univ. Press, New York.

Monaghan, E. P., and Glickman, S. E. (1992). Hormones and aggressive behavior. In J. B. Becker, S. M. Breedlove, and D. Crews (Eds.), Behavioral Endocrinology, pp. 261-285. MIT Press, Cambridge, MA.

Moore, F. L., and Evans, S. J. (1999). Steroid hormones use nongenomic mechanisms to control brain functions and behaviors: A review of evidence. Brain. Behav. Evol. 54(1), 41-50.

Packard, M. G., Schroeder, J. P., and Alexander, G. M. (1998). Expression of testosterone conditioned place preference is blocked by peripheral or intra-accumbens injection of alpha-flupenthixol. Horm. Behav. 34(1), 39-47. 
Rabkin, J. G., Wagner, G., and Rabkin, R. (1996). Treatment of depression in HIV+ men: Literature review and report of an ongoing study of testosterone replacement therapy. Ann. Behav. Med. 18, 24-29.

Reber, A. S. (1989). Implicit learning and tacit knowledge. J. Exp. Psychol. Gen. 118, 219-235.

Reed, J., and Johnson, P. (1994). Assessing implicit learning with indirect tests: Determining what is learned about sequence structure. J. Exp. Psychol. Learning Memory Cognition 20, 585-594.

Riad-Fahmy, D., Read, G. F., Walker, R. F., Walker, S. M., and Griffiths, K. (1987). Determination of ovarian steroid hormone levels in saliva. An overview. J. Reprod. Med. 32(4), 254-272.

Schultheiss, O. C. (2001). An information processing account of implicit motive arousal. In M. L. Maehr and P. Pintrich (Eds.), Advances in Motivation and Achievement, Vol. 12: New Directions in Measures and Methods, pp. 1-41. JAI Press, Greenwich, CT.

Schultheiss, O. C., and Brunstein, J. C. (in press). Inhibited power motivation and persuasive communication: A lens model analysis. J. Pers.

Schultheiss, O. C., Campbell, K. L., and McClelland, D. C. (1999). Implicit power motivation moderates men's testosterone responses to imagined and real dominance success. Horm. Behav. 36(3), 234-241.

Smith, C. P. (Ed.) (1992). Motivation and Personality: Handbook of Thematic Content Analysis. Cambridge University Press, New York.

Suay, F., Salvador, A., Gonzalez-Bono, E., Sanchis, C., Martinez, M., Martinez-Sanchis, S., Simon, V. M., and Montoro, J. B. (1999). Effects of competition and its outcome on serum testosterone, cortisol and prolactin. Psychoneuroendocrinology 24, 551-566.

Winter, D. G. (1973). The Power Motive. Free Press, New York.

Winter, D. G. (1994). Manual for Scoring Motive Imagery in Running Text, 4th ed. Unpublished manuscript, Department of Psychology, University of Michigan, Ann Arbor. 